\title{
Wireless Signaling with Identical Quanta
}

\author{
R. Song (Student Member), C. Rose (Fellow), Y-L Tsai (Student Member), and I. S Mian
}

\begin{abstract}
In this short paper we describe an approach and preliminary results for obtaining bounds on mutual information between release times and capture times for a set of $M$ identical quanta traveling from a source to a target. The first-passage times are assumed independent and identically distributed and the launch times are constrained. One major application of area is intercellular molecular signaling in biological systems whereby a cell (or group of cells) must deliver some message (such as developmental instructions) over distance with reasonable certainty to another cell (or group of cells). Another application area is communication between components of nano/molecular computers. However, the model can also be applied to any communication systems wherein indistinguishable signals have random transit latencies.

Index Terms-nanocommunication, diffusion channel, biological signaling
\end{abstract}

\section{INTRODUCTION}

Biological systems are networks of intercommunicating elements at whatever level one cares to consider (macro)molecules, cells, tissues, organisms, populations, microbiomes, ecosystems, and so on. It is therefore no wonder that communication theorists have plied their trade heavily in this scientific domain (for a recent review, see [1]). Biological systems offer a dizzying array of processes and phenomena through which the same and/or different tasks, communication or otherwise, might be accomplished (see, for example, [2]-[7]). Identifying the underlying mechanisms (signaling modality, signaling agent, signal transport) as well as the molecules and structures implementing the mechanisms is no small undertaking. Consequently, experimental biologists use a combination of prior knowledge and what can only be called instinct to choose those systems on which to expend effort. Guidance may be sought from evolutionary developmental biology - a field that compares the developmental processes of different organisms to determine their ancestral relationship and to discover how developmental processes evolved. Insights may be gained by using statistical machine learning techniques to analyze heterogeneous data such as the biomedical literature and the output of so-called "omics" technologies - genomics (genes, regulatory, and non-coding sequences), transcriptomics (RNA and gene expression), proteomics (protein expression), metabolomics (metabolites and metabolic networks), pharmacogenomics (how genetics affects hosts' responses to drugs), and physiomics (physiological dynamics and functions of whole organisms).

Typically, the application of communication theory to biology starts by selecting a candidate system whose components and operations have already been at least partially elucidated using methods in the experimental and/or computational biology toolkit [8], [9] and then applying communication theoretic methods [1], [7], [10]-[12]. However, we believe that communication theory in general and information theory in particular are not merely system analysis tools for biology. That is, given energy constraints and some general physics of the problem, an information-theoretic treatment can be used to provide outer bounds on information transfer in a mechanism-blind manner. Thus, rather than simply elucidating and quantifying known biology, communication theory can winnow the plethora of possibilies (or even suggest new ones) amenable to experimental and computational pursuit. Likewise, general application of communication-theoretic principles to biology affords a new set of application areas for communication theorists. Some aspects of the potential for communication theory as a new lens on biological systems are explored in [13].

Numerous scenarios in biology that involve the transmission of information can be synthesized and summarized by inscribed matter being sent by an emitter, moving through a medium, and arriving eventually at its destination receptor where it is interpreted [14]. Scenarios illustrating the complexity and diversity that our abstraction attempts to capture include the following:

- messenger RNA molecules (mRNAs) that are transcribed from the genome migrate from the nucleus to the cytoplasm where they are translated by the ribosome into proteins.

- Molecules of the neurotransmitter acetylcholine (Ach) that are released by the presynaptic neuron terminal diffuse through the synaptic cleft and bind to nicotinic Ach receptors on the motor end plate.

- Ions, molecules, organelles, bacteria and viruses that are present in one cell are shipped through a thin membrane channel (tunneling nanotube) to the connected cell where they elicit a physiological response.

- Membrane-bound vesicles that contain a variety of materials and substances translocate through the cytoplasm to the cell membrane where release their contents into the extracellular environment.

- Malignant cells that have escaped the confines of a tissue circulate through the bloodstream to other sites where they re-penetrate the vessel walls and can seed a new tumor. 
- Chemicals factors that are secreted or excreted by an individual travel outside the body where they are sensed by a member of the same species triggering a social or behavioral response.

Our emitter-receptor system is also motivated by fundamental "systems" problems in biology such as development, wherein undifferentiated cells are "told" what to become by a combination of internal programming and extracellular milieu signals - and in turn tell other cells what to become [15]. Thus, communication within and between cells plays a vital role in the development (embryogenesis), maintenance (tissue homeostasis), subversion (disorders such as cancer, inflammation, infections) and decline (aging) of multicellular forms and systems.

Finally, though we have concentrated on biological systems here, the same ideas apply to emerging molecular computing systems wherein "normal" methods of communication (i.e., wires) are either ineffecient or inappropriate. Thus, we here explore an abstraction that encompasses wireless communication systems which use some type of quanta for message passing. Owing to space limitations only results are provided. Proofs will appear in [16].

\section{Problem Definition}

Typically, information is thought to be conveyed via numbers of signaling quanta (concentration). Thus, what amount to dose-response curves are the norm for a variety of experimental biology studies [7] as well as clever theoretical workups (e.g., [17]). However, as was shown in an entirely different domain and unrelated work [18], timing of emissions could in principle also convey information. Clearly, this possibility cannot be ignored if our aim is to attempt to provide bounds on what "a cell can tell the world." Under certain conditions, perhaps timing is a useful complement to concentration. Alternatively, timing might sometimes be energetically unfavorable and its use unlikely. In either case, information-theoretic bounds would help guide biological inquiry.

To begin, consider $M$ quanta launched at times $\left\{T_{m}\right\}$, $m=1,2, \ldots, M$. The launch time ensemble probability density $f_{\mathbf{T}}(\mathbf{t})$ is assumed causal, but otherwise arbitrary. The duration of quantum $m$ 's first-passage between source and destination is $D_{m}$. We assume only that the $D_{m}$ are i.i.d. with $f_{D_{m}}(d)=g(d)=G^{\prime}(d)$ where $g()$ is some causal probability density with mean $\frac{1}{\lambda}$ and Cumulative Distribution Function (CDF) $G()$. We also assume that $g()$ contains no singularities.

Thus, the first portion of the channel is modeled as a sum of random $M$-vectors

$$
\mathbf{S}=\mathbf{T}+\mathbf{D}
$$

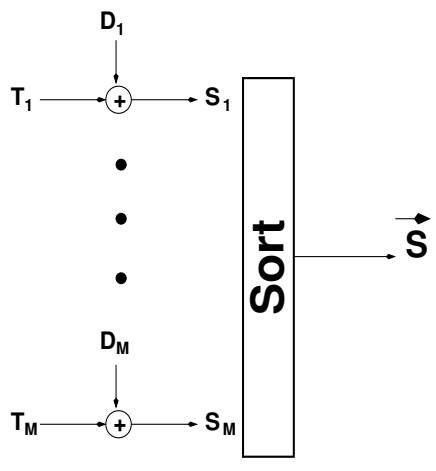

Fig. 1: Quanta release channel with reordering.

for which we have

$$
\begin{aligned}
f_{\mathbf{S}}(\mathbf{s}) & =\int_{0}^{\infty} f_{\mathbf{T}}(\mathbf{t}) f_{\mathbf{S} \mid \mathbf{T}}(\mathbf{s} \mid \mathbf{t}) d \mathbf{t} \\
& =\int_{0}^{\mathbf{s}} f_{\mathbf{T}}(\mathbf{t}) \prod_{m=1}^{M} g\left(s_{m}-t_{m}\right) d \mathbf{t} \\
& =\int_{\mathbf{0}}^{\mathbf{s}} f_{\mathbf{T}}(\mathbf{t}) \mathbf{g}(\mathbf{s}-\mathbf{t}) d \mathbf{t}
\end{aligned}
$$

where

$$
\mathbf{g}(\mathbf{s}-\mathbf{t})=\prod_{m=1}^{M} g\left(s_{m}-t_{m}\right)
$$

With mean constraints on the $T_{m}$, the channel between $\mathbf{T}$ and $\mathbf{S}$ is exactly the parallel version of the model introduced in Bits Through Queues [18]. However, since the molecules are identical, we cannot necessarily determine which arrival corresponds to which launch time. Thus, the final output of the channel is a reordering of the $\left\{s_{m}\right\}$ to obtain a set $\left\{\vec{s}_{m}\right\}$ where $\vec{s}_{m} \leq \vec{s}_{m+1}$, $m=1,2, \ldots, M-1$. (See FIGURE 1.) We write this relationship as

$$
\overrightarrow{\mathbf{S}}=P_{\Omega}(\mathbf{S})
$$

where $P_{k}()$ is a permutation operator and $\Omega$ is that permutation index which produces an ordered $\overrightarrow{\mathbf{S}}$ from the argument $\mathbf{S}$. Incidentally, we define $P_{1}()$ as the identity permutation operator, $P_{1}(\mathbf{s})=\mathbf{s} \forall \mathbf{s}$. We note that the event $S_{i}=S_{j}(i \neq j)$ is of zero measure owing to the no-singularity assumption on $g()$. Thus, for analytic convenience we will assume that $f_{\mathbf{S}}(\mathbf{s})=0$ whenever two or more of the $s_{m}$ are equal and therefore that the $\left\{\vec{s}_{m}\right\}$ are strictly ordered wherever $f_{\overrightarrow{\mathbf{S}}}() \neq 0$ (i.e., $\vec{s}_{m}<\vec{s}_{m+1}$ ).

Thus, the density $f_{\overrightarrow{\mathbf{S}}}(\mathbf{s})$ can be found by "folding" the density $f_{\mathbf{S}}(\mathbf{s})$ about the hyperplanes described by one or more of the $s_{m}$ equal until the resulting probability density is nonzero only on the region where $s_{m}<s_{m+1}$. Analytically we have

$$
f_{\overrightarrow{\mathbf{S}}}(\mathbf{s})= \begin{cases}\sum_{n=1}^{M !} f_{\mathbf{S}}\left(P_{n}(\mathbf{s})\right) & \mathbf{s} \in \mathcal{S}_{1} \\ 0 & \text { otherwise }\end{cases}
$$


where we have defined $\mathcal{S}_{1}$ as the region in s-space for which $s_{1}<s_{2}<\cdots<s_{m}$.

We can likewise describe $f_{\overrightarrow{\mathbf{S}} \mid \mathbf{T}}(\mathbf{s} \mid \mathbf{t})$ as

$$
f_{\overrightarrow{\mathbf{S}} \mid \mathbf{T}}(\mathbf{s} \mid \mathbf{t})= \begin{cases}\sum_{\substack{n=1 \\ 0}}^{M !} f_{\mathbf{S} \mid \mathbf{T}}\left(P_{n}(\mathbf{s}) \mid \mathbf{t}\right) & \mathbf{s} \in \mathcal{S}_{1} \\ & \text { otherwise }\end{cases}
$$

which to emphasize the assumed causality of $g()$ we rewrite as

$$
f_{\overrightarrow{\mathbf{S}} \mid \mathbf{T}}(\mathbf{s} \mid \mathbf{t})=\sum_{n=1}^{M !} \mathbf{g}\left(P_{n}(\mathbf{s})-\mathbf{t}\right) \mathbf{u}\left(P_{n}(\mathbf{s})-\mathbf{t}\right)
$$

where

$$
\mathbf{u}\left(P_{n}(\mathbf{s})-\mathbf{t}\right)=\prod_{m=1}^{M} u\left(\left[P_{n}(\mathbf{s})\right]_{m}-t_{m}\right)
$$

$u()$ is the usual unit step function, and we of course assume $\mathbf{s} \in \mathcal{S}_{1}$.

When $g(d)=\lambda e^{-\lambda d} u(d)$, the conditional distribution on the ordered output $\overrightarrow{\mathbf{S}}$ takes the particularly simple form

$$
f_{\overrightarrow{\mathbf{S}} \mid \mathbf{T}}(\mathbf{s} \mid \mathbf{t})=\lambda^{M} e^{-\lambda \sum_{i=1}^{M}\left(s_{i}-t_{i}\right)}\left(\sum_{n=1}^{M !} \mathbf{u}\left(P_{n}(\mathbf{s})-\mathbf{t}\right)\right)
$$

for $\mathbf{s} \in \mathcal{S}_{1}$. It is worth mentioning explicitly that equation (7) does not assume $s_{i} \geq t_{i}$ as might be implicit in equation (2).

Finally, the following property of expectations of hyper-symmetric functions over hyper-symmetric random variables will later prove useful. The term hypersymmetry is used to emphasize that the function is constant over any permutation of variables. Suppose $Q(\mathbf{x})$ is a hyper-symmetric function and $\mathbf{X}$ is a hypersymmetric random vector. Then, when $\overrightarrow{\mathrm{X}}$ is the ordered version of random vector $\mathbf{X}$ we have

$$
E_{\overrightarrow{\mathbf{X}}}[Q(\overrightarrow{\mathbf{X}})]=E_{\mathbf{X}}[Q(\mathbf{X})]
$$

With these preliminaries done, we can now begin to examine the mutual information between $\mathbf{T}, \mathbf{S}$ and $\overrightarrow{\mathbf{S}}$.

\section{Mutual Information Between T And $\overrightarrow{\mathbf{S}}$}

The mutual information between $\mathbf{T}$ and $\mathbf{S}$ is

$$
I(\mathbf{S} ; \mathbf{T})=h(\mathbf{S})-h(\mathbf{S} \mid \mathbf{T})
$$

Since the $S_{i}$ given the $T_{i}$ are mutually independent, $h(\mathbf{S} \mid \mathbf{T})$ does not depend on $f_{\mathbf{T}}(\mathbf{t})$. Thus, maximization of equation (9) is simply a maximization of the marginal $h(S)$ over the marginal $f_{T}(t)$, a problem explicitly considered and solved for a mean $T_{m}$ constraint in [18] and for a peak constraint in [19].
The corresponding expression for the mutual information between $\mathbf{T}$ and $\overrightarrow{\mathbf{S}}$ is

$$
I(\overrightarrow{\mathbf{S}} ; \mathbf{T})=h(\overrightarrow{\mathbf{S}})-h(\overrightarrow{\mathbf{S}} \mid \mathbf{T})
$$

Unfortunately, $h(\overrightarrow{\mathbf{S}} \mid \mathbf{T})$ now does depend on the input distribution and the optimal form of $h(\overrightarrow{\mathbf{S}})$ is non-obvious. So, rather than attempting a brute force optimization of equation (10) by deriving order distributions [20], we first invoke simplifying symmetries.

Consider that a launch vector $\mathbf{t}$ and any of its permutations $P_{n}(\mathbf{t})$ produce statistically identical outputs $\overrightarrow{\mathbf{S}}$ owing to the reordering operation as depicted FIGURE 1. Thus, any $f_{\mathbf{T}}()$ which optimizes equation (10) can be "balanced" to form an optimizing input distribution which obeys

$$
f_{\mathbf{T}}(\mathbf{t})=f_{\mathbf{T}}\left(P_{n}(\mathbf{t})\right) n=1,2, \cdots, M !
$$

with $P_{n}()$ the previously defined permutation operator. We will therefore restrict our search to "hypersymmetric" densities $f_{\mathbf{T}}(\mathbf{t})$ as defined by equation (11).

If we assume $f_{\mathbf{T}}()$ is hyper-symmetric, then it is easy to show that $f_{\mathbf{S}}()$ must also be hyper-symmetric. From equation (2) we have

$$
f_{\mathbf{S}}\left(P_{n}(\mathbf{s})\right)=\int_{\mathbf{0}}^{P_{n}(\mathbf{s})} f_{\mathbf{T}}(\mathbf{t}) \mathbf{g}\left(P_{n}(\mathbf{s})-\mathbf{t}\right) d \mathbf{t}
$$

If we define $\mathbf{t}^{\prime}=P_{n}^{-1}(\mathbf{t})$ then we can write

$$
\begin{aligned}
f_{\mathbf{S}}\left(P_{n}(\mathbf{s})\right) & =\int_{\mathbf{0}}^{\mathbf{s}} f_{\mathbf{T}}\left(P_{n}^{-1}\left(\mathbf{t}^{\prime}\right)\right) \mathbf{g}\left(\mathbf{s}-\mathbf{t}^{\prime}\right) d \mathbf{t}^{\prime} \\
& =\int_{\mathbf{0}}^{\mathbf{s}} f_{\mathbf{T}}\left(\mathbf{t}^{\prime}\right) \mathbf{g}\left(\mathbf{s}-\mathbf{t}^{\prime}\right) d \mathbf{t}^{\prime} \\
& =f_{\mathbf{S}}(\mathbf{s})
\end{aligned}
$$

The hyper-symmetry of $f_{\mathbf{S}}(\mathbf{s})$ leads to a simple expression for $f_{\overrightarrow{\mathbf{S}}}(\mathbf{s})$. As before we define $\mathcal{S}_{1}$ as the region in s-space for which $s_{1}<s_{2}<\cdots<s_{m}$. Similarly define disjoint regions $\mathcal{S}_{n}$ as those for which if $\mathbf{s} \in \mathcal{S}_{n}$ then $P_{n}(\mathbf{s}) \in \mathcal{S}_{1}$. That is, $\mathcal{S}_{n}$ is the region in s-space in which application of permutation operator $P_{n}()$ orders the components from smallest to largest.

Following equation (4) we have

$$
f_{\overrightarrow{\mathbf{S}}}(\mathbf{s})=M ! f_{\mathbf{S}}(\mathbf{s})
$$

for $\mathbf{s} \in \mathcal{S}_{1}$. We can then write

$$
\begin{aligned}
h(\overrightarrow{\mathbf{S}}) & =-\int_{\mathcal{S}_{1}} M ! f_{\mathbf{S}}(\mathbf{s}) \log \left(M ! f_{\mathbf{S}}(\mathbf{s})\right) d \mathbf{s} \\
& =-M ! \int_{\mathcal{S}_{1}} f_{\mathbf{S}}(\mathbf{s}) \log f_{\mathbf{S}}(\mathbf{s}) d \mathbf{s}-\log M !
\end{aligned}
$$

But since $f_{\mathbf{S}}(\mathbf{s})$ is hyper-symmetric, we also have

$$
h(\overrightarrow{\mathbf{S}})=-\sum_{n=1}^{M !} \int_{\mathcal{S}_{n}} f_{\mathbf{S}}\left(P_{n}(\mathbf{s})\right) \log f_{\mathbf{S}}\left(P_{n}(\mathbf{s})\right) d \mathbf{s}-\log M !
$$

which since $f_{\mathbf{S}}()$ is hyper-symmetric becomes

$$
h(\overrightarrow{\mathbf{S}})=h(\mathbf{S})-\log M !
$$

We state this result as a theorem. 
Theorem 1: If $f_{\mathbf{T}}()$ is a hyper-symmetric probability density function on launch times $\left\{T_{m}\right\}, m=1,2, . ., M$, and $f_{D}()$ is a singularity-free first passage density, then the entropy of the size-ordered outputs $\overrightarrow{\mathbf{S}}$ is

$$
h(\overrightarrow{\mathbf{S}})=h(\mathbf{S})-\log M \text { ! }
$$

Next we turn to $h(\overrightarrow{\mathbf{S}} \mid \mathbf{T})$. As before we define $\Omega$ as the permutation index number that produces an ordered output from $\mathbf{S}$. That is, $P_{\Omega}(\mathbf{S})=\overrightarrow{\mathbf{S}} \in \mathcal{S}_{1}$. Specification of the random tuple $(\Omega, \overrightarrow{\mathbf{S}})$ is equivalent to specifying $\mathbf{S}$ and vice versa. Therefore, we have

$$
h(\mathbf{S} \mid \mathbf{T})=h(\Omega, \overrightarrow{\mathbf{S}} \mid \mathbf{T})=h(\overrightarrow{\mathbf{S}} \mid \mathbf{T})+H(\Omega \mid \overrightarrow{\mathbf{S}}, \mathbf{T})
$$

which we rearrange as

$$
h(\overrightarrow{\mathbf{S}} \mid \mathbf{T})=h(\mathbf{S} \mid \mathbf{T})-H(\Omega \mid \overrightarrow{\mathbf{S}}, \mathbf{T})
$$

$H(\Omega \mid \overrightarrow{\mathbf{S}}, \mathbf{T})$ is the uncertainty about which $S_{m}$ corresponds to which $\vec{S}_{m}$ given both $\mathbf{T}$ and $\overrightarrow{\mathbf{S}}$, and we note that

$$
0 \leq H(\Omega \mid \overrightarrow{\mathbf{S}}, \mathbf{T}) \leq \log M !
$$

We can then write the ordered mutual information as

$$
I(\overrightarrow{\mathbf{S}} ; \mathbf{T})=h(\mathbf{S})-h(\mathbf{S} \mid \mathbf{T})-(\log M !-H(\Omega \mid \overrightarrow{\mathbf{S}}, \mathbf{T}))
$$

after assuming that $f_{\mathbf{T}}()$ is hyper-symmetric.

Since $h(\mathbf{S} \mid \mathbf{T})$ is a constant with respect to $f_{\mathbf{T}}(\mathbf{t})$, maximization of mutual information in equation (15) requires we maximize the expression

$$
h(\mathbf{S})+H(\Omega \mid \overrightarrow{\mathbf{S}}, \mathbf{T})
$$

with respect to $f_{\mathbf{T}}(\mathbf{t})$.

Mutual information is convex in $f_{\mathbf{T}}(\mathbf{t})$ and the space $\mathcal{F}_{\mathbf{T}}$ of feasible hyper-symmetric $f_{\mathbf{T}}(\mathbf{t})$ is convex. Thus, we can in principle apply variational [21] techniques to find that hyper-symmetric $f_{\mathbf{T}}()$ which attains the unique maximum of equation (10). However, in practice, direct application of this method can lead to grossly infeasible $f_{\mathbf{T}}()$, implying that the optimizing $f_{\mathbf{T}}()$ lies in some corner of the convex search space.

Regardless, we must first understand the component parts of the optimization, in particular $H(\Omega \mid \overrightarrow{\mathbf{S}}, \mathbf{T})$ for which we derive expressions and bounds in the next two sections.

$$
\text { IV. } H(\Omega \mid \overrightarrow{\mathbf{S}}, \mathbf{t})
$$

The optimization stated in equation (16) hinges on specification of $H(\Omega \mid \overrightarrow{\mathbf{S}}, \mathbf{T})$ and we first consider $H(\Omega \mid \overrightarrow{\mathbf{s}}, \mathbf{t})$, the admissible-permutation entropy given $\overrightarrow{\mathbf{s}}$ and $\mathbf{t}$. Given $\mathbf{t}$, the probability that $\mathbf{S}$ produced $\overrightarrow{\mathbf{S}}$ is

$$
\operatorname{Prob}(\Omega=k \mid \overrightarrow{\mathbf{s}}, \mathbf{t})=\frac{f_{\mathbf{S} \mid \mathbf{T}}(\overrightarrow{\mathbf{s}} \mid \mathbf{t})}{\sum_{n=1}^{M !} f_{\mathbf{S} \mid \mathbf{T}}\left(P_{n}(\overrightarrow{\mathbf{s}}) \mid \mathbf{t}\right)}
$$

where $\overrightarrow{\mathbf{s}}=P_{\Omega}(\mathbf{s})$. Owing to the causality of $g()$, some permutations will have zero probability since the specific $\overrightarrow{\mathbf{s}}$ and $\mathbf{t}$ may render them impossible via causality.

Using equation (6), the definition of entropy and equation (17) we have

$$
H(\Omega \mid \overrightarrow{\mathbf{s}}, \mathbf{t})=-\sum_{n=1}^{M !} \mathcal{P}_{n}(\overrightarrow{\mathbf{s}}, t) \log \mathcal{P}_{n}(\overrightarrow{\mathbf{s}}, t)
$$

where

$$
\mathcal{P}_{n}(\overrightarrow{\mathbf{s}}, t)=\frac{\mathbf{g}\left(P_{n}(\overrightarrow{\mathbf{s}})-\mathbf{t}\right)}{\sum_{j=1}^{M !} \mathbf{g}\left(P_{j}(\overrightarrow{\mathbf{s}})-\mathbf{t}\right)}
$$

and as might be imagined, equation (18) is difficult to work with in general.

However, for exponential $g()$, we can use equation (7) to simplify equation (17) as

$$
\operatorname{Prob}(\Omega=k \mid \overrightarrow{\mathbf{s}}, \mathbf{t})=\frac{\mathbf{u}(\overrightarrow{\mathbf{s}}-\mathbf{t})}{\sum_{n=1}^{M !} \mathbf{u}\left(P_{n}(\overrightarrow{\mathbf{s}})-\mathbf{t}\right)}
$$

which is a uniform probability mass function with $\sum_{n=1}^{M !} \mathbf{u}\left(P_{n}(\overrightarrow{\mathbf{s}})-\mathbf{t}\right)$ elements. Thus, we can write

$$
H_{e}(\Omega \mid \overrightarrow{\mathbf{s}}, \mathbf{t})=\log \sum_{n=1}^{M !} \mathbf{u}\left(P_{n}(\overrightarrow{\mathbf{s}})-\mathbf{t}\right)
$$

The summation is the number of admissible permutations given $\overrightarrow{\mathbf{s}}$ and $\mathbf{t}$, and constitutes an upper bound for all possible causal first-passage time densities, $g()$. In addition, the exponential first passage time density is the only density which maximizes $H_{e}(\Omega \mid \overrightarrow{\mathbf{s}}, \mathbf{t})$. We state the result as a theorem:

Theorem 2: If we define

$$
|\Omega|_{\overrightarrow{\mathbf{s}}, \mathbf{t}}=\sum_{n=1}^{M !} \mathbf{u}\left(P_{n}(\overrightarrow{\mathbf{s}})-\mathbf{t}\right)
$$

then

$$
H(\Omega \mid \overrightarrow{\mathbf{s}}, \mathbf{t}) \leq \log |\Omega|_{\overrightarrow{\mathbf{s}}, \mathbf{t}}=H_{e}(\Omega \mid \overrightarrow{\mathbf{s}}, \mathbf{t})
$$

with equality iff $g()$ is exponential.

Then, defining

$$
H^{\uparrow}(\mathbf{t}) \equiv E_{\overrightarrow{\mathbf{s}} \mid \mathbf{t}}\left[H_{e}(\Omega \mid \overrightarrow{\mathbf{s}}, \mathbf{t})\right]
$$

we have via Theorem 2:

Theorem 3: Let

$$
H^{\uparrow}(\mathbf{T}) \equiv E_{\overrightarrow{\mathbf{T}}}\left[H^{\uparrow}(\overrightarrow{\mathbf{T}})\right]
$$

then since

$$
H(\Omega \mid \overrightarrow{\mathbf{S}}, \mathbf{t}) \leq H^{\uparrow}(\mathbf{t})
$$

we have

$$
H(\Omega \mid \overrightarrow{\mathbf{S}}, \mathbf{T}) \leq H^{\uparrow}(\mathbf{T})
$$

with equality iff $g()$ is exponential. 


\section{BOUNDS ON $I(\overrightarrow{\mathbf{S}} ; \mathbf{T})$ FOR EXPONENTIAL $g()$}

For exponential first passage, $H(\Omega \mid \overrightarrow{\mathbf{S}}, \mathbf{T})=H^{\uparrow}(\mathbf{T})$ via Theorem 3. However, the $f_{\mathbf{T}}()$ which maximizes $h(\mathbf{s})+H(\Omega \mid \mathbf{T}, \overrightarrow{\mathbf{S}})$ has proved elusive. We therefore calculated $H(\Omega \mid \mathbf{T}, \overrightarrow{\mathbf{S}})$ for i.i.d. launch time densities which maximize $h(\mathbf{S})$ alone under mean launch time and launch deadline constraints as derived in [19]. The result was the following two bounds for $I(\overrightarrow{\mathbf{S}} ; \mathbf{T})$.

Theorem 4: If $E\left[T_{m}\right] \leq \tau, m=1,2, \ldots, M$, then

$$
\begin{aligned}
M \log (1+\lambda \tau) & \geq I(\overrightarrow{\mathbf{S}} ; \mathbf{T}) \\
& \geq M \log (1+\lambda \tau)-\log M ! \\
& +\sum_{k=0}^{M} \log (k !)\left(\begin{array}{c}
M \\
k
\end{array}\right) p^{M-k}(1-p)^{k-1}
\end{aligned}
$$

where

$$
p=\frac{\lambda \tau}{\lambda \tau+1}
$$

Theorem 5: If $T_{m} \in[0, \tau], m=1,2, \ldots, M$, then

$$
\begin{aligned}
M \log \left(1+\frac{\lambda \tau}{e}\right) & \geq I(\overrightarrow{\mathbf{S}} ; \mathbf{T}) \\
& \geq M \log \left(1+\frac{\lambda \tau}{e}\right)-\log M ! \\
& +\sum_{\ell=1}^{M-1} \Delta \Gamma_{M \ell} \log (\ell+1) !
\end{aligned}
$$

where

$$
\begin{aligned}
& \Delta \Gamma_{M, \ell}=\lambda \tau\left(\begin{array}{c}
M \\
\ell+1
\end{array}\right)\left(\frac{1}{e+\lambda \tau}\right)^{M} \\
& \times\left[\begin{array}{c}
\ell+1 \\
e^{\ell+1}(\lambda)^{M-\ell-2} \\
+ \\
(e+\lambda \tau-1)^{M-\ell-2}((\ell+1)(e+\lambda \tau)-M)
\end{array}\right]
\end{aligned}
$$

In both theorems, the first inequality follows from the data processing inequality [22].

\section{Discussion AND CONCLUSION}

We have derived bounds on mutual information between launch times $\mathbf{T}$ and reception times $\overrightarrow{\mathbf{S}}$ for $M$ identical signaling quanta. While previous work focused on models derived from brownian motion and required numerical calculation [20], here we assumed arbitrary causal densities and side-stepped output order distribution derivations on $\overrightarrow{\mathbf{S}}$ by; (a) using hyper-symmetric input densities which result in a simple expression for output entropy $h(\overrightarrow{\mathbf{S}})$, (b) decomposition of $I(\overrightarrow{\mathbf{S}} ; \mathbf{T})$ into a sum of $I(\vec{S} ; \mathbf{T})$ and $H(\Omega \mid \overrightarrow{\mathbf{S}}, \mathbf{T})$, the output order permutation entropy given the input and output, and (c) deriving an upper bound $H^{\uparrow}(\mathbf{T}) \geq H(\Omega \mid \overrightarrow{\mathbf{S}}, \mathbf{T})$ valid for any causal first passage density. Tight upper bounds $I(\overrightarrow{\mathbf{S}} ; \mathbf{T})$ via maxmization of equation (15) eluded us, so the data processing theorem was employed. Lower bounds were obtained by using entropy-maximizing $f_{\mathbf{T}}()$ [19] and evaluating $H^{\uparrow}(\mathbf{T})$. The methods described here undergird upcoming work on the bounds of diffusive molecular communication in biological systems [16].

\section{REFERENCES}

[1] O. Milenkovic, G. Alterovitz, G. Battail, T. P. Coleman, J. Hagenauer, S. P. Meyn, N. Price, M. F. Ramoni, I. Shmulevich, and W. Szpankowski. Introduction to the special issue on information theory in molecular biology and neuroscience. Trans. Information Theory, 56(2):649-652, 2010.

[2] C. de Joussineau, J. Soule, M. Martin, C Anguille, P. Montcourrier, and D. Alexandre. Delta-Promoted Filopodia Mediate Long-Range Lateral Inhibition in Drosophila. Nature, 426:555559, December 42003.

[3] Y.A. Gorby, S. Yanina, JS.. McLean, K.M. Rosso, D. Moyles, A. Dohnalkova, T.J. Beveridge, I.S. Chang, B.H. Kim, K.S. Kim, D.E. Culley, S.B. Reed, M.F. Romine, D.A. Saffarini, E.A. Hill, L. Shi, D.A. Elias, D.W. Kennedy, G. Pinchuk, K. Watanabe, S. Ishii, B. Logan, K.H. Nealson, and J.K. Fredrickson. Electrically conductive bacterial nanowires produced by Shewanella oneidensis strain MR-1 and other microorganisms. Proc Natl Acad Sci U.S.A., 103:11358-11363, 2006.

[4] S. Gurke, J.F.V. Barroso, and H.-H. Gerdes. The art of cellular communication: tunneling nanotubes bridge the divide. Histochem Cell Biol, 129:539-550, 2008.

[5] X. Wang, M.L. Veruki, N.V. Bukoreshtliev, E. Hartveit, and H.-H. Gerdes. Animal cells connected by nanotubes can be electrically coupled through interposed gap-junction channels. Proc Natl Acad Sci USA, 107:17194-17199, 2010.

[6] H.C. Berg and E.W. Purcell. Physics of Chemoreception. Biophysical Journal, 20:193-219, 1977.

[7] Pankaj Mehta et al. Information processing and signal integration in bacterial quorum sensing. Molecular systems biology, 2009.

[8] A.L. Hodgkin and A.F. Huxley. A quantitative description of membrane current and its application to conduction and excitation in nerve. J. Physiol., 117(4):500-544, 1952.

[9] Tao Long et al. Quantifying the integration of quorum-sensing signals with single-cell resolution. Molecular systems biology, 2009.

[10] Elek Wajnryb Jose M. Amigo, Janusz Szczepanski and Maria V. Sanchez-Vives. Estimating the entropy rate of spike trains via lempel-ziv complexity. Neural Computation, 16:717-736, 2004.

[11] D.H. Johnson. Information Theory and Neural Information Processing. Trans. Information Theory, 56(2):653-666, Feb 2010.

[12] Riccardo Barbieri, Loren M. Frank, David P. Nguyen, Michael C. Quirk, Victor Solo, Matthew A. Wilson, and Emery N. Brown. Dynamic analyses of information encoding in neural ensembles. Neural Computation, 16:277-307, 2004.

[13] I.S. Mian and C. Rose. Communication theory and multicellular biology. Integrative Biology, 3(4):350-367, April 2011.

[14] C. Rose and G. Wright. Inscribed Matter As An Energy-Efficient Means Of Communication With An Extraterrestrial Civilization. Nature, 431:47-49, 2004.

[15] C. Nusslein-Volhard. Coming to Life: how genes drive development. Kales Press, 2006.

[16] Y-L Tsai, C. Rose, R. Song, and S. Mian. Channels with Multiple Identical Quanta: mutual information bounds. IEEE Trans. on Info. Th., October 2011. (in preparation).

[17] A. Einolghozati, M. Sardari, A. Beirami, and F. Fekri. Capacity of Discrete Molecular Diffusion Channels. In IEEE International Symposium on Information Theory (ISIT) 2011, pages 603-607, July 2011. ISBN: 978-1-4577-0594-6.

[18] V. Anantharam and S. Verdu. Bits Through Queues. IEEE Transactions on Information Theory, 42(1):4-18, January 1996.

[19] Y-L Tsai, C. Rose, R. Song, and S. Mian. An Additive Exponential Noise Channel with a Transmission Deadline. In IEEE International Symposium on Information Theory (ISIT) 2011, pages 598-602, July 2011. ISBN: 978-1-4577-0594-6.

[20] A.W. Eckford. Nanoscale communication with Brownian motion. In CISS'07, pages 160-165, 2007. Baltimore.

[21] F.B. Hildebrand. Advanced Calculus for Applications. Prentice Hall, Englewood Cliffs, NJ, 1976.

[22] T.M. Cover and J.A. Thomas. Elements of Information Theory. Wiley-Interscience, 1991. 